MITSUBISHI ELECTRIC RESEARCH LABORATORIES

http://www.merl.com

\title{
Phase Noise-Robust LLR Calculation with Linear/Bilinear Transform for LDPC-Coded Coherent Communications
}

\author{
Koike-Akino, T.; Millar, D.S.; Kojima, K.; Parsons, K.
}

TR2015-039 May 2015

\begin{abstract}
We propose a modified log-likelihood ratio (LLR) calculation for an LDPC decoder to be robust against residual phase noise at the demodulator. The proposed scheme is based on a linear/bilinear transform offers 1-2dB gain in the presence of large phase noise.

Conference on Lasers and Electro-Optics (CLEO)
\end{abstract}

This work may not be copied or reproduced in whole or in part for any commercial purpose. Permission to copy in whole or in part without payment of fee is granted for nonprofit educational and research purposes provided that all such whole or partial copies include the following: a notice that such copying is by permission of Mitsubishi Electric Research Laboratories, Inc.; an acknowledgment of the authors and individual contributions to the work; and all applicable portions of the copyright notice. Copying, reproduction, or republishing for any other purpose shall require a license with payment of fee to Mitsubishi Electric Research Laboratories, Inc. All rights reserved.

Copyright (C) Mitsubishi Electric Research Laboratories, Inc., 2015

201 Broadway, Cambridge, Massachusetts 02139 



\title{
Phase Noise-Robust LLR Calculation with Linear/Bilinear Transform for LDPC-Coded Coherent Communications
}

\author{
Toshiaki Koike-Akino, David S. Millar, Keisuke Kojima, Kieran Parsons \\ Mitsubishi Electric Research Labs. (MERL), 201 Broadway, Cambridge, MA 02139, USA. $\quad$ koike@merl.com
}

\begin{abstract}
We propose a modified log-likelihood ratio (LLR) calculation for an LDPC decoder to be robust against residual phase noise at the demodulator. The proposed scheme is based on a linear/bilinear transform offers $1 \sim 2 \mathrm{~dB}$ gain in the presence of large phase noise.

OCIS codes: (060.4510) Optical communications, (060.1660) Coherent communications, (060.4080) Modulation.
\end{abstract}

\section{Introduction}

Thanks to the recent advancement of powerful forward-error correction codes, such as low-density parity-check (LDPC) codes, achieving capacity is no longer idealistic especially for additive white Gaussian noise channels. However, optical communications can suffer from various other impairments such as Kerr nonlinearity. To cope with these impairments, the so-called turbo principle [1-8] has drawn much attention. For example, Djordjevic et al. have investigated turbo equalization to mitigate linear and nonlinear distortions [1,2]. In an analogous context, data-dependent second-order statistics of nonlinear distortion has been considered in sliding-window turbo equalizers [3,4]. Wu et al. studied turbo carrier-phase recovery [5]. Turbo differential decoding [6] has been used to mitigate error propagation in differential encoding. Cycle slip issues for blind carrier-phase estimators have been dealt with by turbo slip recovery [7] using a hidden Markov model. Also, transmitter angular skew has been compensated by turbo skew recovery [8].

In particular for such turbo receivers, log-likelihood ratio (LLR) calculation at the demodulator plays an important role for LDPC decoder to work properly. In this paper, we propose a modified LLR calculation in the presence of residual phase noise at the demodulator. Phase noise has been one of the major impairments in optical communications. For example, laser linewidth [9] and fiber nonlinearity [10] can cause stochastic phase noise. In [11], phase noise-robust constellation design has been studied. Recently, Cao et al. considered a phase noise-aware LLR calculation based on a modified Bessel function for orthogonal frequency-division multiplexing (OFDM) [12].

In this paper, we adopt linear and bilinear transforms to derive an alternative closed-form LLR formula, and show its benefit in LDPC-coded single-carrier systems using high-order quadrature-amplitude modulation (QAM) formats.

\section{Linear-Transformed (LT) and Bilinear-Transformed (BLT) LLR Calculation Robust to Phase Noise}

In practice, any carrier-phase recovery method is imperfect, and a certain amount of residual phase noise will remain at a demodulator before LLR calculation. We consider the signal model as follows

$$
R=\mathrm{e}^{\mathrm{j} \theta} S+N, \quad \theta \sim \mathbb{N}\left(0, \sigma_{\mathrm{p}}^{2}\right), \quad N \sim \mathbb{C} \mathbb{N}\left(0, \sigma^{2}\right),
$$

where $R$ is the received symbol at the demodulator, $S$ is the transmitted QAM symbol, $\theta$ is the residual phase noise, which follows the Gaussian distribution $\mathbb{N}(\cdot)$ of zero mean and $\sigma_{\mathrm{p}}^{2}$ variance, and $N$ is an additive noise following the circular-symmetry complex Gaussian distribution $\mathbb{C N}(\cdot)$ of zero mean and $\sigma^{2}$ variance. The likelihood becomes

$$
\operatorname{Pr}(R \mid S)=\int \operatorname{Pr}(R \mid S, \theta) \operatorname{Pr}(\theta) \mathrm{d} \theta=\int \frac{1}{\pi \sigma^{2}} \mathrm{e}^{-\frac{1}{\sigma^{2}}\left|R-\mathrm{e}^{\mathrm{j} \theta} S\right|^{2}} \frac{1}{\sqrt{2 \pi \sigma_{\mathrm{p}}^{2}}} \mathrm{e}^{-\frac{\theta^{2}}{2 \sigma_{\mathrm{P}}^{2}}} \mathrm{~d} \theta,
$$

where $\operatorname{Pr}(\cdot)$ denotes probability. To obtain a closed-form solution of the equation, we introduce the linear transform (LT), i.e., $\mathrm{e}^{\mathrm{j} \theta} \simeq 1+\mathrm{j} \theta$, or bilinear transform (BLT), i.e., $\mathrm{e}^{\mathrm{j} \theta} \simeq \frac{1+\mathrm{j} \theta / 2}{1-\mathrm{j} \theta / 2}$. With LT, the log-likelihood is expressed as

$$
\log \operatorname{Pr}(R \mid S)=-\frac{1}{\sigma^{2}}|R-S|^{2}+\frac{2 \sigma_{\mathrm{p}}^{2} / \sigma^{2}}{\sigma^{2}+2 \sigma_{\mathrm{p}}^{2}|S|^{2}}\left(\mathfrak{I}\left[S^{*} R\right]\right)^{2}-\frac{1}{2} \log \left(\sigma^{2}+2 \sigma_{\mathrm{p}}^{2}|S|^{2}\right)+\text { c.c },
$$

where $\mathfrak{I}[\cdot],[\cdot]^{*}$, and c.c denote the imaginary part, complex conjugate, and constant coefficient, respectively. The first term is a conventional log-likelihood formula, while the other terms modify the LLR calculation by taking the phase noise into account. For BLT, we just need to replace $S$ with $(R+S) / 2$, and $R$ with $(3 R-S) / 2$ in the above equation. 


\section{Performance Results}

Here, we show the benefit of the modified LLR calculation in the presence of residual phase noise. Figs. 1(a) and 1(b) show post-LDPC bit-error-rate (BER) performance of 4QAM and 1024QAM, respectively. We use an irregular LDPC code, whose codeword length is 38400 and code rate is 0.8 . For 4QAM, we consider a large phase noise variance of $\sigma_{\mathrm{p}}^{2}=0.128$. It is shown that the modified LLR calculations with LT/BLT offers more than 0.8dB gain compared to the conventional LLR calculation at a BER of $3 \times 10^{-3}$. For this case, BLT is $0.25 \mathrm{~dB}$ better than LT. For 1024QAM, we consider a phase noise variance of $\sigma_{\mathrm{p}}^{2}=0.002$. The LT-LLR outperforms the conventional LLR by $2.2 \mathrm{~dB}$. We did not present BLT performance because there was no performance improvement compared with LT. Instead, we plot the performance of 8-iteration turbo demodulation $[7,8]$ with LT-LLR, which achieves an additional $1 \mathrm{~dB}$ gain.

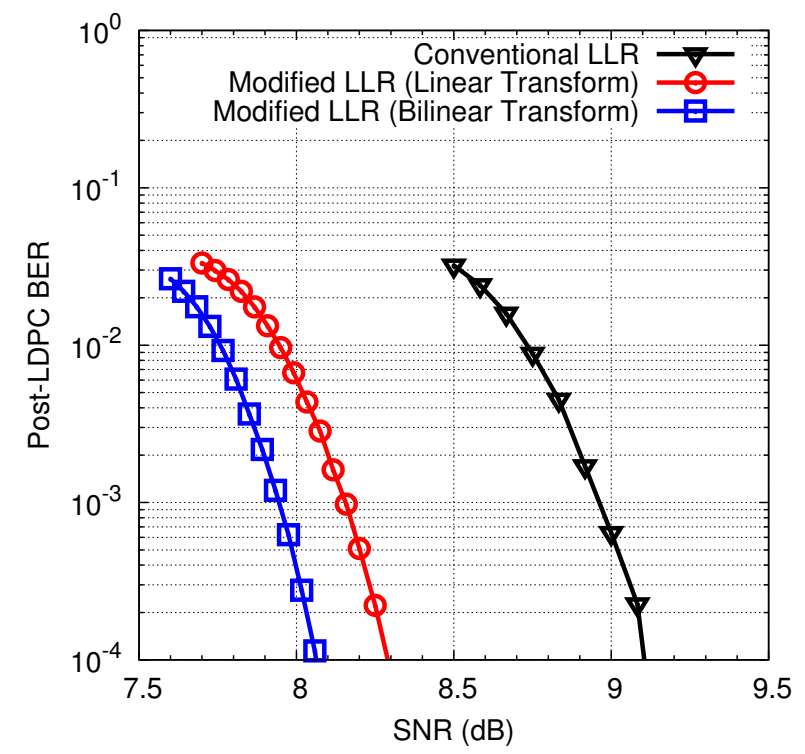

(a) 4QAM $\left(\sigma_{\mathrm{p}}^{2}=0.128\right)$

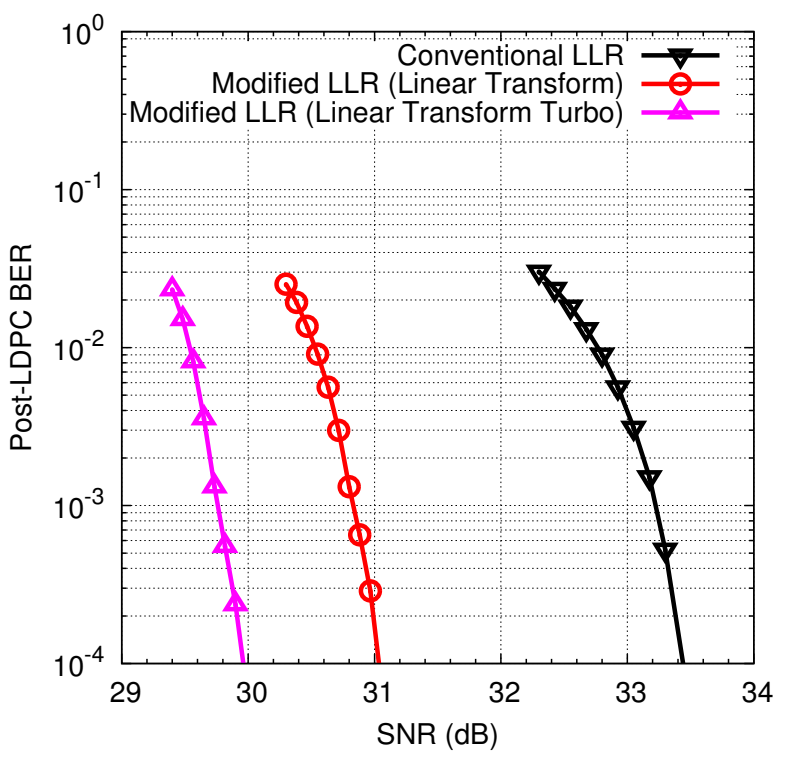

(b) 1024QAM $\left(\sigma_{\mathrm{p}}^{2}=0.002\right)$

Fig. 1: Post-LDPC BER performance of linear/bilinear-transformed LLR calculation.

\section{Conclusions}

We have proposed modified closed-form LLR formulas based on linear/bilinear transforms for an LDPC decoder to be robust against residual phase noise at demodulator. The proposed method showed a significant gain of $1 \sim 2 \mathrm{~dB}$ in the presence of large phase noise for high-order QAM transmissions. An additional gain provided by turbo demodulation in conjunction with the modified LLR was also evaluated.

\section{References}

1. I.B. Djordjevic, L. L. Minkov, H. G. Batshon, IEEE JSAC 266 (2008): 73-83.

2. I.B. Djordjevic, L. Lyubomir, L. Minkov, L. Xu, T. Wang, IEEE/OSA J. Opt. Commun. Netw. 16 (2009): 555-564.

3. C. Duan, et al., $O F C$, JW2A.5 (2012)

4. T. Fujimori et al., OECC, ThR2.3 (2013)

5. X. Wu, H. Xiang, IEEE commun. lett. 98 (2005): 735-737.

6. F. Yu et al., ECOC, We.10.P1.70 (2011)

7. T. Koike-Akino, K. Kojima, D.S. Millar, K. Parsons, OFC M3A.3 (2014)

8. T. Koike-Akino, K. Kojima, D.S. Millar, K. Parsons, ECOC Th.1.3.2 (2014)

9. K. Kikuchi, Optics Express 205 (2012): 5291-5302.

10. A.P.T. Lau, J.M. Kahn, IEEE JLT 25 10, 3008-3016 (2007)

11. L. Beygi, E. Agrell, M. Karlsson, OFC, OThO4 (2011)

12. S. Cao et al., IEEE PTL 2615 (2014): 1577-1580. 